\title{
Prevalência de Toxocaríase e Fatores Associados em Crianças de uma Escola Pública em Belo Horizonte, Minas Gerais, Brasil
}

\author{
Seroprevalence for Toxocariasis between Schoolchildren of a \\ Public School in Belo Horizonte, Minas Gerais, Brazil and its \\ Associated Factors
}

Recebido em: 21/11/2016 Aceito em: $18 / 04 / 2017$
Ludmilla Parreiras CAMPOS ${ }^{1}$; Elaine Alvarenga de Almeida CARVALHO ${ }^{1}$; Gabriela de Moraes SOARES ${ }^{2}$; Júnea CHAGAS $^{1}$; Kênia da Silva COSTA ${ }^{1}$; Edna Afonso REIS ${ }^{1}$; Carlos James SCAINI' ${ }^{2}$; Micheline Rosa SILVEIRA ${ }^{1}$ ${ }^{1}$ Universidade Federal de Minas Gerais. Avenida Presidente Antônio Carlos, 6627, Pampulha, CEP 31270-901, Belo Horizonte, MG, Brasil. ${ }^{2}$ Universidade Federal do Rio Grande. Rua Visconde de Paranaguá, 102, Centro, CEP 96203-900. Rio Grande, RS, Brasil. E-mail: lucamp23@gmail.com

\section{ABSTRACT}

A high prevalence of toxocariasis has been observed in many countries worldwide, especially in tropical countries. The present study aimed to estimate the prevalence of toxocariasis and its associated factors in 100 children from a public school in the city of Belo Horizonte, Brazil. Sociodemographic data, lifestyle habits, self-reported health conditions, as well as characteristics related to housing and the presence of pets, were collected. In addition, clinical exams, anthropometric measurements, and laboratory exams (hemogram, total IgG, total IgE, and anti-Toxocara canis $\operatorname{IgG}$ ELISA) were carried out. Among the evaluated children, 15\% presented positive serology for toxocariasis. The participants with positive and negative results were compared by means of either a Student's t test, for the two samples, or the Mann-Whitney test, and only the total IgG parameter proved to be statistically different among them. Considering the positive serology, the variable of age, the presence of puppies, contact with the ground, and the BMI (thinness) status proved to be significantly associated. These variables were included in a multiple logistics regression model to identify the factors independently associated with the response variable. The significance level adopted in the final model was of 0.05 . Also in the final model, the variable of contact with the ground and the BMI status remained at a p-value of 0.025 and 0.030 , respectively. An epidemiologically relevant prevalence of toxocariasis was found in the studied population. This prevalence serves as a warning sign that disease control measures need to be better publicized and implemented.

Keywords: children; zoonoses; seroepidemiologic studies; toxocariasis

\section{RESUMO}

Tem sido demostrada uma alta prevalência de toxocaríase em diferentes países do mundo, principalmente nos países tropicais. Dessa forma o objetivo desse estudo foi estimar a prevalência de toxocaríase e seus fatores associados em cem crianças de uma escola pública de Belo Horizonte, Brasil. Foram coletadas informações sociodemográficas, hábitos de estilo de vida, condições de saúde autorreferida, características relacionadas à moradia e à presença de animais domésticos. Também foram realizados exames clínicos, medidas antropométricas e exames laboratoriais (hemograma, IgG total, IgE total e ELISA para detecção de anticorpos IgG anti-Toxocara canis). Entre as crianças avaliadas, 
$15 \%$ apresentaram sorologia positiva para toxocaríase. Os participantes com resultados positivos e os negativos foram comparados por teste T para duas amostras ou Teste de Mann-Whitney e apenas o parâmetro IgG total foi estatisticamente diferente entre eles. Entre os avaliados com sorologia positiva, as variáveis idade, presença de filhotes, contato com terra e status de IMC (magreza) mostraram-se significativamente associadas. Essas variáveis foram incluídas em um modelo de regressão logística múltiplo, para identificar os fatores associados, de forma independente, com a variável resposta. O nível de significância adotado no modelo final foi de 0,05 . Permaneceram no modelo final o contato com terra e o status de IMC com valores p 0,025 e 0,030, respectivamente. Uma prevalência epidemiologicamente significativa de toxocaríase foi encontrada na população estudada, o que serve como alerta para que medidas de controle da doença sejam divulgadas e implementadas.

Palavras-Chave: criança; zoonoses; estudos soroepidemiológicos; toxocaríase.

\section{INTRODUÇÃO}

A toxocaríase é uma zoonose de distribuição mundial, primeiramente descrita por Beaver (1952) (1), causada por Toxocara canis e Toxocara cati, parasitas intestinais de cães e gatos, respectivamente. Os humanos são acometidos quando ingerem acidentalmente ovos contendo a larva no estágio L2 (2). A doença pode ocorrer em decorrência da migração das larvas e da liberação do antígeno de secreção e excreção (TES), que permite a sobrevivência da larva e é altamente imunogênico, provocando reação imune, principalmente do tipo Th2 (3). As manifestações podem acontecer sob a forma de cinco tipos de síndromes: Larva Migrans Visceral (LMV), Larva Migrans Ocular (LMO), Larva Migrans Neurológica (LMN), Toxocaríase Oculta e Toxocaríase Assintomática (4), as quais apresentam diferentes manifestações clínicas e laboratoriais.

A prevalência em humanos tem se mostrado variável. Na cidade de Pelotas, Rio Grande do Sul, Brasil, $50,6 \%$ das crianças apresentaram o Teste de Imunodiagnóstico ELISA (Enzyme Linked Immunoabsorbent Assay) positivo (5), resultado semelhante ao encontrado em escolares em Vitória, Espírito Santo, Brasil (6). Nos Estados Unidos, uma prevalência de $13,9 \%$ foi encontrada em indivíduos com idade superior a seis anos (7); na Croácia $31 \%$ de crianças com eosinofilia apresentaram ELISA positivo para $T$. canis (8); na Dinamarca foi reportada uma prevalência de $2,4 \%$ (9), e na ilha de La Reunion, 92,8\% (10). O estudo americano de Won e cols. (2008), correlacionou a sorologia positiva a baixa educação, pobreza, altas concentrações sanguíneas de chumbo e posse de cão (7), assim como um estudo realizado em Salvador, Bahia, Brasil, em que a prevalência sorológica foi mais alta em indivíduos de classe social baixa, com maior contato com cães e gatos (11). Um outro estudo brasileiro, na cidade de Presidente Prudente,
São Paulo, encontrou uma prevalência geral de sorologia positiva igual a $11,1 \%$ e comparou crianças de classe média (prevalência 9,5\%) e crianças de baixa renda (prevalência $12,7 \%$ ). A alta renda familiar e ser criança do sexo feminino foram considerados fatores de proteção para a população total e para ambos os grupos. Possuir gato foi considerado fator de risco para a população total e para ambos os grupos e possuir cão demostrou ser um fator de risco apenas para crianças de classe média (12).

Estudos demonstraram altas taxas de contaminação em ambientes públicos, como praças e parques, com ovos provenientes das fezes de cães parasitados (13-17), o que faz com que essa doença seja de grande importância para a saúde pública.

O diagnóstico da toxocaríase é baseado na detecção de anticorpos IgG pelo método ELISA, utilizando antígenos TES, $(18,19)$, e o resultado deve ser, preferencialmente, confirmado pelo teste de Western Blot (20). A sorologia ocupa papel importante, uma vez que o diagnóstico definitivo, que é feito pela observação das larvas nos tecidos por meio de biopsia, expõe o paciente a riscos e incômodos; e após alguns anos de infecção, se torna mais difícil a detecção das larvas nos tecidos $(21,22)$. Os pacientes sintomáticos, apresentam, normalmente, além da sorologia positiva, alterações laboratorais como eosinofilia e níveis de IgE aumentados (4, 23-26).

Dessa forma, o objetivo desse estudo foi estimar a prevalência de toxocaríase e seus fatores associados em crianças de uma escola pública de Belo Horizonte, Brasil.

\section{MÉTODO}

Amostra de Estudo. A amostra do estudo foi constituída de 100 crianças, com idade entre sete e 14 anos, de um total de 346 matriculadao, em 2013, do segundo 
ao quinto ano do Ensino Fundamental, em uma escola pública estadual de Belo Horizonte, Minas Gerais, cujos responsáveis assinaram o Termo de Consentimento Livre e Esclarecido (TCLE). Para o cálculo do tamanho mínimo da amostra na estimação de prevalência, foi considerada a população infinita, prevalência presumida de no máximo $20 \%$, nível de confiança de $95 \%$, efeito do desenho igual a 1 e margem de erro máxima de $10 \%$, sendo obtido o tamanho mínimo de 62 crianças. Com a amostra efetivamente coletada de 100 crianças, a margem de erro foi reduzida a 7,84\%, nas mesmas condições.

Obtenção de dados e exames realizados. No período de maio a agosto de 2013, foram realizadas as coletas de sangue para os exames laboratoriais das 100 crianças, sendo esses: hemograma, dosagem de $\operatorname{IgG}$ e IgE e sorologia anti-Toxocara canis, além de entrevistas para o preenchimento de um questionário semiestruturado com questões abertas, para a obtenção de informações referentes às características sociodemográficas, estilo de vida, condições de saúde autorreferida, características relacionadas à moradia e à presença de animais domésticos. Nesse mesmo instrumento também foram preenchidas informações referentes ao exame clínico e medidas antropométricas realizadas pela equipe médica.

As crianças foram avaliadas em relação às medidas antropométricas peso (utilizando uma balança digital) e altura (fita métrica). Com o objetivo de diagnosticar déficits ponderais e/ou estaturais, foi utilizada a calculadora antropométrica do programa computacional WHO ANTHROPIUS, que permite avaliação, em percentil e escore $Z$ - valores e gráficos - de indivíduos de 5 a 19 anos. O peso e a altura foram classificados em: "muito baixo", "baixo", "adequado" e "elevado" para a idade. Em relação ao índice de massa corporal (IMC), que mede a relação entre peso e altura, foram utilizados os seguintes status para os valores de escore Z: "magreza acentuada", "magreza", "eutrófico", "sobrepeso", “obesidade" e "obeso grave".

O hemograma foi realizado por modo automatizado. A dosagem de IgG foi realizada por imunoturbidimetria e a dosagem de IgE por quimioluminescência. Anticorpos IgG específicos anti-Toxocara foram detectados pelo teste ELISA que utiliza antígenos TES originários de larvas de segundo estágio. Os soros teste e os controles positivos e negativos foram pré adsorvidos com extratos de Ascaris summ, para evitar reações cruzadas, e em seguida foram testados na diluição $1: 50 \mathrm{em}$ duplicata em PBS pH 7,2 contendo tween-20 a 0,05\%
(PBS-T). Tanto os soros quanto o conjugado (IgG anti-humano ligado a peroxidase - Sigma $\left.{ }^{\circledR}\right)$ foram incubados durante 45 minutos a $37^{\circ} \mathrm{C}$ e, entre as fases, as placas foram lavadas três vezes com PBS-T, durante 3 minutos. Todos os reagentes foram usados no volume de $100 \mu \mathrm{L}$ por poço. Como cromógeno foi utilizada a ortofenilenodiamina (OPD), na concentração $0,4 \mathrm{mg} / \mathrm{mL}$, em tampão citrato - fosfato $\mathrm{pH} 4,0$, acrescida de peróxido de hidrogênio $0,1 \%$, tendo sido realizada a leitura em $450 \mathrm{~nm}, 15$ minutos após a adição do OPD. O cut off foi estabelecido pela média de leitura óptica de dez crianças consideradas soro negativas acrescida de dois desvios - padrão.

Aspectos Éticos. O estudo foi aprovado pelo Comitê de Ética em Pesquisa da Universidade Federal de Minas Gerais (COEP-UFMG) sob o $\mathrm{n}^{\circ}$ COEP ETIC 0583.0.203.000-10, em 02 de maio de 2011. O TCLE entregue aos responsáveis estava de acordo com Resolução 196/96 (vigente à época) tendo sido solicitada assinatura dos responsáveis e das crianças. Somente foram coletados dados e material biológico das crianças cujos responsáveis assinaram o TCLE. Com o objetivo de cumprir os requisitos éticos, os nomes das crianças foram substituídos por um identificador único na base de dados para garantir a confidencialidade.

Estatística. A variável resposta considerada foi a sorologia positiva para $T$. canis e as variáveis explicativas investigadas foram idade, número de crianças e adultos na residência, presença de cães e cães filhotes, contato com terra, geofagia, onicofagia, alteração de visão, status do IMC, linfadenomegalia superficial, níveis de IgG total, IgE total, contagem global de leucócitos, eosinófilos, hematócrito e hemoglobina.

A análise estatística foi realizada no programa computacional Minitab versão 15.1.1.0. Inicialmente, foi realizada uma análise descritiva dos dados, que incluiu descrição da população estudada, cálculo de distribuições de frequência das variáveis categóricas e medidas de síntese para as variáveis quantitativas. A fim de verificar a existência associação entre sorologia positiva e as variáveis explicativas, foi realizada análise univariada via teste Qui-quadrado ou o teste exato de Fisher. As variáveis que apresentaram um valor de $\mathrm{p}$ menor que 0,20 na análise univariada e que, biologicamente ou epidemiologicamente, eram plausíveis, foram examinadas simultaneamente em um modelo de regressão logística, para identificar os fatores associados com a variável resposta. O nível de significância adotado para a permanência da variável no modelo final foi de 0,05 . 


\section{RESULTADOS E DISCUSSÃO}

As 100 crianças estudadas apresentaram idade média de 9,1 anos, peso médio de $34,9 \mathrm{~kg}$, estatura média de $140 \mathrm{~cm}$, e IMC médio de $17,9 \mathrm{~kg} / \mathrm{m}^{2}$.

Quanto ao status de IMC, nenhuma criança foi classificada com magreza acentuada, quatro tiveram status magreza, 77 como eutróficas, 13 como sobrepeso, cinco como obesidade e apenas uma recebeu classificação obeso grave. Os parâmetros laboratoriais estudados apresentaram os resultados mostrados na Tabela 1.

$\mathrm{O}$ teste ELISA qualitativo anti-Toxocara canis apresentou resultado positivo em 15 crianças e negativo nas demais 85 crianças. Desse modo, a prevalência de toxocaríase desta população foi estimada em $15 \%$ (IC95\%: 8-22\%).

Foi feita uma comparação dos dados laboratoriais entre os grupos com sorologia positiva $(\mathrm{n}=15)$ e sorologia negativa $(n=85)$ com a realização de teste $T$ para duas amostras ou o teste de Mann-Whitney (quando a distribuição da variável no grupo não era Normal), a fim de verificar a existência de diferenças laboratoriais entre eles, como demostrado na Tabela 2.
Essa análise mostrou que os grupos de participantes positivos e negativos são diferentes estatisticamente apenas na dosagem de IgG total, com média estatisticamente maior nos positivos.

A relação da variável resposta (sorologia positiva para $T$. canis) com as variáveis explicativas que foram categorizadas, podem ser visualizadas na Tabela 3 . Apenas mostraram valor $p$ menor que 0,20 as varíaveis: idade ( $<10$ anos), presença de cães filhotes, contato com terra e status de IMC (magreza). As variáveis que apresentaram um valor de $p<0,20$ na análise univariada foram examinadas simultaneamente por meio da regressão logística, para identificar os fatores associados de forma independente com a variável resposta. O nível de significância adotado foi de 0,05 .

Após essa análise, as variáveis que permaneceram no modelo final foram: contato com terra e status de IMC magreza, com valores p de 0,025 e 0,030 respectivamente (Tabela 4).

A presença de animais de estimação, principalmente cães e gatos, em um número cada vez maior de domicílios e o fato de esses animais, tanto os domesticados quanto os errantes, frequentarem locais utilizados por

Tabela 1. Estatísticas descritivas dos resultados dos parâmetros laboratoriais estudados em 100 crianças de uma escola pública de Belo Horizonte, MG, Brasil, 2013.

\begin{tabular}{lcccccccc}
\multicolumn{1}{c}{ Parâmetro } & Média & DP & Mínimo & Máximo & P25 & P50 & P75 \\
\hline Hemoglobina $(\mathrm{g} / \mathrm{dL})$ & 12,81 & 0,84 & 10,60 & 15,00 & 12,20 & 12,800 & 13,300 \\
\hline IgG total $(\mathrm{mg} / \mathrm{dL})$ & 1075,5 & 203,8 & 654,0 & 1566,0 & 931,5 & 1071,0 & 1566,0 \\
\hline IgE total $(\mathrm{UI} / \mathrm{mL})$ & 332,73 & 468,09 & 4,70 & 2491,50 & 37,70 & 128,60 & 405,40 \\
Global de Leucócitos $\left(/ \mathrm{mm}^{3}\right)$ & 7514,0 & 2390,4 & 3600,0 & 20700,0 & 6025,0 & 7150,0 & 8775,0 \\
\hline Eosinófilos $\left(/ \mathrm{mm}^{3}\right)$ & 411,4 & 314,4 & 51,0 & 2100,0 & 185,7 & 315,5 & 575,5 \\
Hematócrito $(\%)$ & 37,99 & 2,41 & 32,70 & 43,90 & 36,10 & 37,95 & 39,80
\end{tabular}

Tabela 2. Valores de média e mediana, valores p para comparação de média ou mediana e intervalo de confiança para a diferença de média ou mediana entre os grupos de sorologia positiva $(n=15)$ e negativa $(n=85)$ de cem crianças de uma escola pública de Belo Horizonte, MG, Brasil, 2013.

\begin{tabular}{|c|c|c|c|c|c|c|}
\hline \multirow{2}{*}{ Parâmetro } & \multicolumn{2}{|c|}{ Sorologia + $(n=15)$} & \multicolumn{2}{|c|}{ Sorologia $-(n=85)$} & \multirow{2}{*}{ Valor p } & \multirow{2}{*}{ IC } \\
\hline & Média & Mediana & Média & Mediana & & \\
\hline Hemoglobina $(\mathrm{g} / \mathrm{dL})$ & 12,95 & 13,1 & 12,78 & 12,70 & 0,59 & $-0,470 ; 0,801$ \\
\hline IgG total $(\mathrm{mg} / \mathrm{dL})$ & 1177 & 1229 & 1057 & 1031 & 0,015 & 25,$3 ; 216,5$ \\
\hline IgE total $(U I / m L)$ & 319,3 & 223,8 & 335,9 & 102,6 & $0,37 * *$ & $-53,4 ; 168,0 * *$ \\
\hline Global de Leucócitos (/ mm³) & 6660 & 6400 & 7620 & 7200 & $0,18 * *$ & $-1999,8 ; 300,1^{* *}$ \\
\hline Eosinófilos $\left(/ \mathrm{mm}^{3}\right)$ & 386,8 & 277,0 & 411,97 & 316,0 & $0,75^{* *}$ & $-139,9 ; 100,0$ ** \\
\hline Hematócrito (\%) & 38,58 & 38,1 & 37,89 & 37,7 & 0,41 & $-1,040 ; 2,424$ \\
\hline
\end{tabular}

** Teste Não Paramétrico Mann - Whitney: valor p e Intervalo de Confiança (IC) para diferenças de Mediana 


\section{luitarma}

humanos para atividades de lazer, tornam a população mais exposta à toxocaríase (27). A infecção pode ocorrer principalmente se os indivíduos não praticarem, frequentemente, a lavagem correta das mãos e alimentos, e se não procederem com a vermifugação em frequência adequada de seus animais de estimação.

Crianças de 1 a 15 anos são consideradas grupo de risco para toxocaríase. devido a um contato muito próximo com animais de estimação infectados e à exposição a ovos embrionados presentes no ambiente (28). Alonso e cols (2000) acharam prevalência maior de toxocaríase em crianças entre 11 e 12 anos (29) e Cilla e cols (1996) mostraram que crianças de 6 a 10 anos tinham maior risco de contrair a doença (30). Entretanto, alguns trabalhos não encontraram a idade como um fator importante para a doença $(31,32)$. No presente trabalho, ter idade menor que dez anos teve valor de $p$ menor que 0,20 na análise univariada, mas não foi significativo no modelo de regressão logística final.

Estudos que analisaram fatores de risco mostraram uma associação entre onicofagia $(6,33)$, geofagia $(24,34,35)$ e presença de cão no domicílio $(7,35-47)$ com a doença. Esses fatores não foram estatisticamente significativos no presente trabalho. Entretanto, os dados

Tabela 3. Análise univariada dos fatores associados com a sorologia anti-Toxocara canis de crianças de uma escola pública de Belo Horizonte, MG, Brasil, 2013 ( $n=100)$

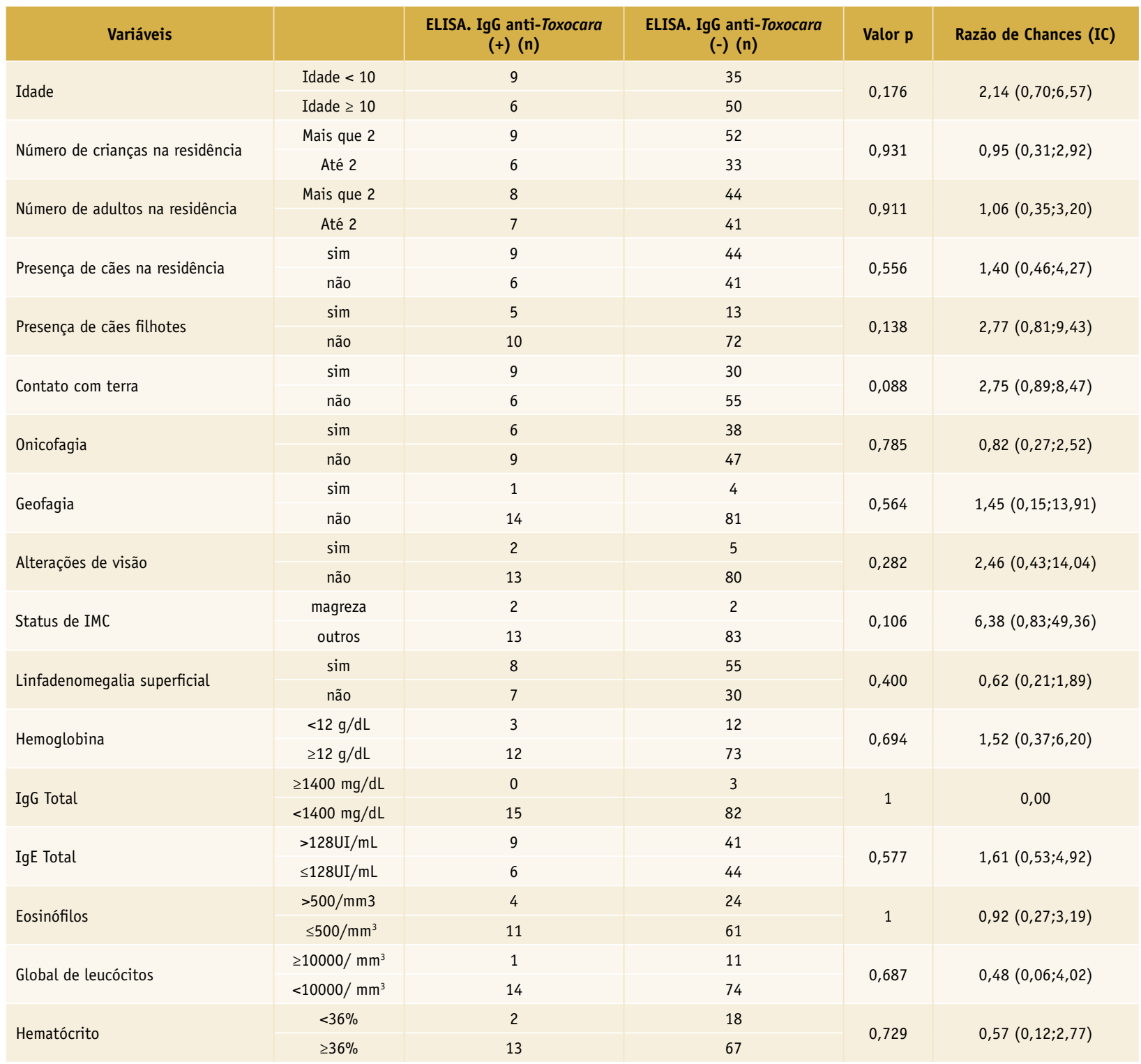

Valor p: teste Qui Quadrado de Pearson/ Teste Exato de Fisher 
encontrados concordam com outros estudos que demostraram que onicofagia $(48)$, geofagia $(39,40,48,49)$ e cão no domicilio $(38,50-53)$ não estavam associados com a toxocaríase. Esses dados evidenciam que ainda existem discordâncias sobre os fatores de risco.

Ter cães filhotes, fator associado com a doença em alguns trabalhos $(43,48)$, apresentou valor de $\mathrm{p}$ menor que 0,20 na análise univariada, mas não foi estatisticamente significativo no modelo final de regressão logística. Mesmo não tendo permanecido no modelo final, parece que esse fator é de grande importância para a infecção. Em relação ao contato com terra, foi encontrada associação estatisticamente significativa com a sorologia positiva (valor p 0,025 , no modelo final de regressão logística), mostrando ser um fator de risco para a doença, dado que concorda com aqueles de Figueiredo e cols. (2005) (48). Devido ao fato de crianças frequentemente terem contato com terra durante suas atividades de lazer em praças, parques ou até mesmo nos quintais de suas casas, esse é um fator de risco que merece muita atenção. A associação da doença com baixa renda também foi discutida e comprovada em alguns trabalhos $(7,11,12,54,55)$ mas não foi analisada no presente estudo.

Foi identificada uma prevalência de $15 \%$ nos escolares avaliados, dado bastante parecido com um outro estudo recente no Brasil, no Estado de São Paulo, em que a prevalência encontrada foi de 15,5\% (34). No entanto, esses dados mostram prevalência menor que outros estudos brasileiros em crianças, que detectaram prevalência de 36,8\% (56), 50,6\% (5) e 51,6 (6). Devido ao fato de as populações estudadas serem diferentes, existe uma dificuldade em comparar esses resultados. No presente trabalho foram avaliadas crianças assintomáticas, estudantes de uma escola pública, com idade entre sete e 14 anos. Mattia e cols (2012) avaliaram crianças com uma faixa etária diferente, sendo essas de zero a 12 anos, atendidas no sistema público de saúde (56). Cassenote e cols. (2014) incluiram crianças de um a 12 anos, provenientes de escolas públicas, filantrópicas e particulares (34); Schoenardie e cols (2013), também estudaram crianças entre um e 12 anos (5), mas utilizaram amostras de uma soroteca, não sendo possível saber, nesse último caso, sobre as condições clínicas e sociodemográficas das crianças estudadas.

No mundo, várias taxas de prevalência de toxocaríase já foram descritas, como $38,8 \%$ em crianças em Cuba (57), 13,9\% em indivíduos maiores que 6 anos nos EUA (7), 11\% em crianças na Holanda (58), 2,1\% em crianças de um a sete anos na Alemanha (59), e 60\% em crianças de cinco a 15 anos de uma comunidade de Santa Lucia no Caribe (60).
A questão nutricional foi abordada e foi um dos fatores associados significativamente com a sorologia positiva (valor $\mathrm{p} 0,030$ no modelo final), mas não pode ser afirmado se o status de IMC de magreza foi um fator de risco para a infecção ou se foi uma consequência desta. A perda de peso (43) e a desnutrição pregressa (48) foram citadas como fatores associados à sorologia positiva para T. canis. Mas em outro trabalho (42) as medidas de crescimento e nutrição não foram diferentes entre os grupos de positivos e negativos. Mais estudos que investiguem a relação entre a toxocaríase e os déficits ponderais são necessários.

Tabela 4. Análise multivariada dos fatores associados com a sorologia anti-Toxocara canis de crianças de uma escola pública de Belo Horizonte, MG, Brasil, 2013 ( $n=100)$.

\begin{tabular}{|cccc|}
\hline Variável & Valor $\mathbf{p}$ & $\begin{array}{c}\text { Razão de } \\
\text { chances }\end{array}$ & IC \\
\hline $\begin{array}{c}\text { Contato com } \\
\text { terra }\end{array}$ & 0,025 & 3,98 & 1,$13 ; 14,01$ \\
\hline Status IMC & 0,030 & 13,25 & 1,$46 ; 120,52$ \\
\hline
\end{tabular}

Eosinofilia $(21,24,29,41,48,61-63)$ e dosagens altas de $\operatorname{IgE}(29,41,46,48,62,63)$ muitas vezes foram associadas a doença, e estão presentes principalmente nos casos sintomáticos. No presente estudo, das 15 crianças positivas, $60 \%$ (9) apresentaram $\operatorname{IgE}$ alterado e $26,67 \%$ (4) apresentaram eosinófilos acima de $500 / \mathrm{mm}^{3}$. No entanto, esses dois parâmetros não foram diferentes estatisticamente entre o grupo dos positivos e dos negativos e também não foram associados à sorologia positiva na análise univariada.

A eosinofilia também não foi estatisticamente associada em alguns estudos $(6,12,38,39,46,64)$. Isso parece se dever ao fato de terem sido avaliadas crianças predominantemente assintomáticas e também ao fato de que esses parâmetros são inespecíficos, e podem ter seus valores alterados em outras condições, como alergias e parasitoses intestinais.

A dosagem de IgG total foi diferente estatisticamente entre os positivos e negativos (valor $\mathrm{p} 0,015$ no teste $\mathrm{T}$ ), tendo os positivos uma média mais alta de $\operatorname{IgG}$ que os negativos, mas o fato de ter IgG total alterado não foi associado à sorologia positiva na análise univariada. Glickman e cols (1979) também demonstraram a associação entre elevação de IgG total e sorologia positiva (65).

É possivel perceber que é muito importante a conscientização da população e dos profissionais de saúde 
sobre a doença e suas formas de prevenção, visto que a prevalência encontrada é significativa. O simples hábito de lavar as mãos diminui as chances de se contaminar e foi estatisticamente associado ao decréscimo da prevalência (34). A correta vermifugação de cães e gatos, tanto filhotes quanto animais adultos é de extrema importância, pois a presença de animais não parasitados contribui significativamente para a soronegatividade em crianças (14). A vermifugação de filhotes deve ocorrer com dois, quatro, seis e oito semanas de idade, e depois mensalmente até os seis meses; sendo que em gatos ela pode começar com três semanas (66). Nos animais adultos, o esquema recomendado é de, em média, quatro vezes ao ano, dependendo do estágio de vida do animal (67).

\section{REFERÊNCIAS}

1. Beaver PC, Snyder CH, Carrera GM, Dent JH, Lafferty JW. Chronic eosinophilia due to visceral larva migrans Report of three cases. Pediatrics. 1952;9(1):7-19.

2. Araujo P. Findings related to the 1st ecdysis of Ascaris lumbricoides, A. suum and Toxocara canis larvae. Rev Inst Med Trop Sao Paulo. 1972;14(2): 83-90.

3. Pinelli E, Aranzamendi C. Toxocara infection and its association with allergic manifestations. Endocr Metab Immune Disord Drug Targets. 2012;12(1):33-44. DOI: $10.2174 / 187153012799278956$

4. Magnaval J, Dorchies P, Morassin B. Actualités de la toxocarose humaine. Pyrexie. 2000;4: 111-5.

5. Schoenardie ER, Scaini CJ, Brod CS, Pepe MS, Villela MM, McBride A, Borsuk S, Berne ME. Seroprevalence of Toxocara infection in children from southern Brazil. J Parasitol. 2013; 99(3):537-9. DOI: 10.1645/ GE-3182.

6. Fragoso RP, Monteiro MBM, Lemos EM, Pereira FEL. Anti-Toxocara antibodies detected in children attending elementary school in Vitoria, State of Espírito Santo, Brazil: prevalence and associated factors. Rev Soc Bras Med Trop. 2011;44(4):461-6. DOI: 10.1590/S003786822011000400012 .

7. Won KY, Kruszon-Moran D, Schantz PM, Jones JL. National seroprevalence and risk factors for zoonotic Toxocara spp. infection. Am J Trop Med Hyg. 2008;79(4):5527. DOI: 10.4269 /ajtmh.2008.79.552

8. Sviben M, Čavlek TV, Missoni EM, Galinović GM. Seroprevalence of Toxocara canis infection among asymptomatic children with eosinophilia in Croatia. J Helminthol. 2009;83(4):369-71. DOI: 10.1017/S0022149X09381213

9. Stensvold CR, Skov J, Møller LN, Jensen PM, Kapel CM, Petersen E, Nielsen HV. Seroprevalence of human toxocariasis in Denmark. Clin Vaccine Immunol. 2009;16(9):1372-3. DOI: 10.1128/CVI.00234-09

\section{CONCLUSÃO}

A prevalência significativa de toxocaríase foi encontrada na população estudada. O contato com terra foi considerado fator de risco para a toxocaríase, e a magreza também foi associada à sorologia positiva, porém não foi possível afirmar se é uma condição que favorece a infecção ou se ocorre posteriormente a ela.

\section{AGRADECIMENTOS}

A todas as crianças participantes e seus pais.

10. Magnaval JF, Michault A, Calon N, Charlet JP. Epidemiology of human toxocariasis in La Reunion. Trans R Soc Trop Med Hyg. 1994;88(5): 531-3. DOI: 10.1016/00359203(94)90148-1

11. Souza RF, Dattoli VCC, Mendonça LR, Jesus JRD, Baqueiro T, Santana CDC, Santos NM, Barrouin-Melo SM, Alcântara-Neves, NM. Prevalence and risk factors of human infection by Toxocara canis in Salvador, State of Bahia, Brazil. Rev Soc Bras Med Trop. 2011;44(4):5169. DOI: $10.1590 / \mathrm{S} 0037-8682201100040002$

12. Santarém VA, Leli FNC, Rubinsky-Elefant G, Giuffrida R. Protective and risk factors for toxocariasis in children from two different social classes of Brazil. Rev Inst Med Trop Sao Paulo. 2011; 53(2):66-72. DOI: 10.1590/ S0036-46652011000200002

13. Santarem VA, Pereira VC, Alegre BC. Contamination of public parks in Presidente Prudente (Sao Paulo, Brazil) by Toxocara spp. eggs. Rev Bras Parasitol Vet. 2012;21(3):323-5. DOI: 10.1590/S198429612012000300029

14. Manini MP, Marchioro AA, Colli CM, Nishi L, Falavigna-Guilherme AL. Association between contamination of public squares and seropositivity for Toxocara spp. in children. Vet Parasitol. 2012;188(1):48-52. DOI: 10.1016/j.vetpar.2012.03.011

15. Santos NM, Silva VMGD, Santos ABD, Souza TPD. Contaminação das praias por parasitos caninos de importância zoonótica na orla da parte alta da cidade de Salvador-Ba. Rev Ciências Medicas Biol. 2010;5(1). DOI: 10.9771/cmbio.v5i1.4579

16. Dumenigo B, Gálvez D. Contaminación de suelos en Ciudad de La Habana con huevos de Toxocara canis. Rev Cubana Med Trop. 1995;47(3):178-80.

17. Laird Pérez RM, Carballo Arrieta D, Reyes Zamora EM, García Roche R, Prieto Díaz V. Toxocara sp. en parques y 
zonas públicas de Ciudad de la Habana. Rev Cubana Hig Epidemiol. 1995;38(2):112-6.

18. De Savigny DH, Voller A, Woodruff AW. Toxocariasis: serological diagnosis by enzyme immunoassay. J Clin Pathol . 1979;32(3):284-8.

19. Bach Rizzati BC. Desenvolvimento do Teste Imunoenzimático, ELISA, para o Diagnóstico da Toxocaríase Humana [dissertação]. São Paulo (SP): Faculdade de Ciências Farmacêuticas Universidade de São Paulo;1984.

20. Fillaux J, Magnaval J-F. Laboratory diagnosis of human toxocariasis. Vet Parasitol. 2013;193(4):327-36. DOI: 10.1016/j.vetpar.2012.12.028

21. Magnaval JF, Glickman LT, Dorchies P, Morassin B. Highlights of human toxocariasis. Korean J Parasitol. 2001;39(1):1-11. DOI:10.3347/kjp.2001.39.1.1

22. Carvalho EAA, Rocha RL. Visceral Larva Migrans Syndromes Associated with Toxocariasis: Epidemiology, Clinical and Laboratory Aspects of Human Toxocariasis. Curr Trop Med Rep. 2014;1(1):74-9. DOI: 10.1007/ s40475-013-0011-6

23. Glickman LT, Schantz PM. Epidemiology and pathogenesis of zoonotic toxocariasis. Epidemiol Rev. 1981;3:230-50.

24. Garcia, LS. Diagnostic medical parasitology. Whashinton D.C: American Society for Microbiology Press;2006

25. Buijs J, Egbers MW, Lokhorst WH, Savelkoul HF, Nijkamp FP. Toxocara-induced eosinophilic inflammation: airway function and effect of anti-IL-5. Am J Respir Crit Care Med. 1995;151(3_pt_1):873-8.

26. Łuzna-Lyskov A. Toxocarosis in children living in a highly contaminated area: an epidemiological and clinical study. Acta Parasitol. 2000;45(1):40-42.

27. Gennari SM, de Jesus Pena HF, Cortez, A. Ocorrência de protozoários e helmintos em amostras de fezes de cães e gatos da cidade de São Paulo. Braz J Vet Res Anim Sci. 1999;36(2):87-91. DOI: 10.1590/S141395961999000200006

28. Fernando SD, Wickramasinghe VP, Kapilananda GMG, Devasurendra RL. Epidemiological aspects and risk factors of toxocariasis in a pediatric population in Sri Lanka. Southeast Asian J Trop Med Public Health. 2007;38(6):983-90.

29. Alonso JM, Bojanich MV, Chamorro M, Gorodner JO. Toxocara seroprevalence in children from a subtropical city in Argentina. Rev Inst Med Trop Sao Paulo. 2000;42(4):235-7. DOI: $10.1590 / \mathrm{S} 0036-$ 46652000000400010

30. Cilla G, Pérez-Trallero E, Gutiérrez C, Part C, Gomáriz M. Seroprevalence of Toxocara infection in middle-class and disadvantaged children in northern Spain (Gipuzkoa, Basque Country). Eur J Epidemiol. 1996;12(5):541-543. DOI: 10.1007/BF00144010

31. Herrmann N, Glickman LT, Schantz PM, Weston MG, Domanski LM. Seroprevalence of zoonotic toxocaria- sis in the United States: 1971-1973. Am J Epidemiol. 1985;122(5):890-6. DOI: 10.1093/oxfordjournals.aje. a114172

32. Ljungström I, Van Knapen F. An epidemiological and serological study of Toxocara infection in Sweden. Scand J Infect Dis. 1989;21(1):87-93. DOI: 10.3109/00365548909035685

33. Alderete J, Jacob C, Pastorino AC, Elefant GR, Castro AP, Fomin AB, Chieffi PP. Prevalence of Toxocara infection in schoolchildren from the Butantã region, São Paulo, Brazil. Mem Inst Oswaldo cruz. 2003;98(5):5937.

34. Cassenote AJF, Abreu Lima AR, Pinto Neto JM, Rubinsky-Elefant G. Seroprevalence and Modifiable Risk Factors for Toxocara spp. in Brazilian School children. PLoS Negl Trop Dis. 2014;8(5):e2830. DOI: 10.1371/ journal.pntd.0002830

35. Holland CV, O'Lorcain P, Taylor MRH, Kelly A.Sero-epidemiology of toxocariasis in school children. Parasitology. 1995; 110 (5):535-45. DOI: 10.1017/ S0031182000065252

36. Oliart-Guzmán H, Delfino BM, Martins AC, Mantovani SA, Braña AM, Pereira TM, Branco FL, Ramalho AA, Campos RG, Fontoura PS, et al. Epidemiology and Control of Child Toxocariasis in the Western Brazilian Amazon-A Population-Based Study. Am J Trop Med Hyg. 2014;90(4):670-81. DOI: 10.4269/ajtmh.13-0506

37. Żarnowska H, Borecka A, Gawor J, Marczyńska M, Dobosz S, Basiak W. A serological and epidemiological evaluation of risk factors for toxocariasis in children in central Poland. J Helminthol. 2008;82(2):123-7. DOI: 10.1017/S0022149X08912372

38. Anaruma Filho F, Chieffi PP, Correa CRS, Camargo ED, Silveira EP, Aranha JJB, Ribeiro MC. Human toxocariasis: a seroepidemiological survey in the municipality of Campinas (SP), Brazil. Rev Inst Med Trop Sao Paulo. 2002;44(6):303-7. DOI: 10.1590/ S0036-46652002000600002

39. Glickman LT, Chaudry IU, Costantino J, Clack FB, Cypess RH, Winslow L. Pica patterns, toxocariasis, and elevated blood lead in children. Am J Trop Med Hyg. 1981;30(1):77-80. DOI: 10.4269/ajtmh.1981.30.77

40. Coelho LM, Silva MV, Dini CY, Giacon Neto AA, Novo NF, Silveira EP. Human toxocariasis: a seroepidemiological survey in schoolchildren of Sorocaba, Brazil. Mem Inst Oswaldo Cruz. 2004;99(6):533-57. DOI: 10.1590/ S0074-02762004000600002

41. Marmor M, Glickman L, Shofer F, Faich LA., Rosenberg C, Cornblatt B, Friedman S. Toxocara canis infection of children: epidemiologic and neuropsychologic findings. Am J Public Health. 1987;77(5):554-9. DOI: 10.2105/AJPH.77.5.554

42. Ellis GS, Pakalnis VA, Worley G, Green JA, Frothingham TE, Sturner RA, Walls KW. Toxocara canis in- 
festation: clinical and epidemiological associations with seropositivity in kindergarten children.Ophthalmology. 1986;93(8):1032-7. DOI: 10.1016/S0161-6420(86)33625-X

43. Iddawela DR, Kumarasiri PV, Wijesundera MS. A seroepidemiological study of toxocariasis and risk factors for infection in children in Sri Lanka. Southeast Asian J Trop Med Public Health. 2003;34(1):7-15.

44. Souza FA. Parâmetros clínicos laboratoriais na evolução de 104 crianças portadoras de larva migrans visceral por Toxocara canis [dissertação]. São Paulo (SP): Universidade Federal de São Paulo; 1992.

45. Schantz PM, Meyer D, Glickman LT. Clinical, serologic, and epidemiologic characteristics of ocular toxocariasis. Am J Trop Med Hyg. 1979;28(1):24-8. DOI: 10.4269/ajtmh.1979.28.24

46. Gonzalez-Quintela A, Gude F, Campos J, Garea MT, Romero PA, Rey J, Meijide LM, Fernandez-Merino MC, Vidal C. Toxocara infection seroprevalence and its relationship with atopic features in a general adult population. Int Arch Allergy Immunol. 2005;139(4):317-24. DOI: $10.1159 / 000091603$

47. Fan CK, Liao CW, Kao TC, Li MH, Du WY, Su, KE. Sero-epidemiology of Toxocara canis infection among aboriginal schoolchildren in the mountainous areas of north-eastern Taiwan. Ann Trop Med Parasitol. 2005;99(6):593-600. DOI: 10.1179/136485905X51373

48. Figueiredo SD, Taddei JAAC, Menezes JJ, Novo NF, Silva EO, Cristóvão HL, Cury MC. Estudo clínico-epidemiológico da toxocaríase em população infantil. J Pediatr. 2005;81(2):126-132.

49. Lopez ML, Martin G, Chamorro MC, Mario AJ. Toxocariasis in children from a subtropical region. Medicina. 2004;65(3):226-230.

50. Agudelo C, Villareal E, Cáceres E, López C, Eljach J, Ramírez, Hernandez C, Corredor A. Human and dogs Toxocara canis infection in a poor neighborhood in Bogota. Mem Inst Oswaldo Cruz. 1990;85(1):75-8. DOI: 10.1590/S0074-02761990000100012

51. Genchi C, Di Sacco B, Gatti S, Sangalli G, Scaglia M. Epidemiology of human toxocariasis in northern Italy. Parasitologia. 1990;32(3)313-19.

52. Buijs J, Borsboom G, van Gemund JJ, Hazebroek A, van Dongen PA, van Knapen F, Neijens HJ. Toxocara seroprevalence in 5-year-old elementary schoolchildren: relation with allergic asthma. Am J Epidemiol. 1994;140(9):83947. DOI: 10.1093/oxfordjournals.aje.a117332

53. Ajayi OO, Duhlinska DD, Agwale SM, Njoku M. Frequency of human toxocariasis in Jos, Plateau state, Nigeria. Mem Inst Oswaldo Cruz. 2000;95(2):147-9. DOI: 10.1590/S0074-02762000000200002.

54. Schantz PM. Toxocara larva migrans now. Am J Trop Med Hyg. 1989;41(3 Part 2):21-34. DOI: 10.4269/ajtmh.1989.41.21
55. Campos Júnior D, Elefant GR, Silva EODM, Gandolfi L, Jacob CMA, Tofeti A, Pratesi R. Frequency of seropositivity to Toxocara canis in children of different socioeconomic strata. Rev Soc Bras Med Trop. 2003;36(4):50913. DOI: $10.1590 / \mathrm{S} 0037-86822003000400013$

56. Mattia S, Colli CM, Adami CM, Guilherme GF, Nishi L, Rubinsky-Elefant G, et al. Seroprevalence of Toxocara infection in children and environmental contamination of urban areas in Paraná State, Brazil. J Helminthol. 2012;86(4):440. DOI: 10.1017/S0022149X11000666

57. Sariego I, Kanobana K, Junco R, Vereecken K, Núñez FA, Polman K, Bonet M, Rojas L. Frequency of antibodies to Toxocara in Cuban schoolchildren. Trop Med Int Health. 2012;17(6):711-14. DOI: 10.1111/j. 1365-3156.2012.02996.x

58. Van Gemund JJ, Buijs J, Van Dongen PA, Van den Bergh JP. Seroprevalence of Toxocara infection in young children in the city of The Hague. Trop Geogr Med. 1989;41(4):294-6.

59. Kimmig P, Naser K, Frank W. Seroepidemiologic studies of human toxocariasis. Zentralbl Hyg Umweltmed. 1991;191(4):406-22.

60. Bundy DA, Thompson DE, Robertson BD, Cooper ES. Age-relationships of Toxocara canis seropositivity and geohelminth infection prevalence in two communities in St. Lucia, West Indies. Trop Med Parasitol. 1987;38,(4):309-12.

61. PawlowskI Z. Toxocariasis in humans: clinical expression and treatment dilemma. J Helminthol. 2001;75(4):299305. DOI: 10.1017/S0022149X01000464

62. Glickman LT, Magnaval JF, Domanski LM, Shofer FS, Lauria SS, Gottstein B, Brochier B. Visceral larva migrans in French adults: a new disease syndrome?. Am J Epidemiol. 1987;125(6):1019-34. DOI: 10.1093/oxfordjournals.aje.a114618

63. Buijs J, Egbers M W, Nijkamp FP. Toxocara canis-induced airway hyporeactivity in mice. Agents Actions Suppl. 1989;31:75-80.

64. Gueglio B, De Gentile L, Nguyen JM, Achard J, Chabasse D, Marjolet M. Epidemiologic approach to human toxocariasis in western France. Parasitology Res. 1994;80(6):531-536. DOI: 10.1007/BF00932703

65. Glickman LT, Schantz PM, Cypess RH. Epidemiological characteristics and clinical findings in patients with serologically proven toxocariasis. Trans R Soc Trop Med Hyg. 1979;73(3):254-8. DOI: 10.1016/00359203(79)90077-4

66. Worm Control in Dogs and Cats Guideline 01. ESCCAP (Europen Counsel for Companion Animal Parasites). 2010; $2^{\text {nd }}$ ed: 5-6. Available from: www.esccap.org

67. Overgaauw PAM, Van Knapen F. Veterinary and public health aspects of Toxocara spp. Vet Parasitol. 2013;193(4):398-403. DOI: 10.1016/j.vetpar.2012.12.035 